\title{
BMJ Open Acupuncture for biliary colic: a systematic review protocol
}

\author{
Ning Sun (D) , 'Wenwei Zuo, ${ }^{2}$ Yuanfang Zhou (D) , ${ }^{1}$ Ying Cheng, ${ }^{1}$ Shirui Cheng, ${ }^{1}$ \\ Jun Zhou, ${ }^{1}$ Guixing Xu, ${ }^{1}$ Liuyang Huang, ${ }^{1}$ Fanrong Liang (D) , Ruirui Sun (D) ${ }^{1}$
}

To cite: Sun N, Zuo W, Zhou Y, et al. Acupuncture for biliary colic: a systematic review protocol. BMJ Open 2021;11:e041931. doi:10.1136/ bmjopen-2020-041931

- Prepublication history for this paper is available online. To view these files, please visit the journal online (http://dx.doi. org/10.1136/bmjopen-2020041931).

NS, WZ and YZ are joint first authors.

Received 23 June 2020 Revised 09 December 2020 Accepted 29 December 2020

Check for updates

(c) Author(s) (or their employer(s)) 2021. Re-use permitted under CC BY-NC. No commercial re-use. See rights and permissions. Published by BMJ.

${ }^{1}$ Acupuncture and Tuina School/ The 3rd Teaching Hospital, Chengdu University of Traditional Chinese Medicine, Chengdu,

China

${ }^{2}$ Department of General Surgery, The First Affiliated Hospital of Chengdu University of Traditional Chinese Medicine, Chengdu, China

Correspondence to

Dr Ruirui Sun;

sunruirui@cdutcm.edu.cn and

Dr Fanrong Liang;

acuresearch@126.com

\section{ABSTRACT}

Introduction Biliary colic $(\mathrm{BC})$ is a severe pain associated with nausea and vomiting, which is the most common symptom among the gallstone population. This protocol proposes a methodology for conducting a systematic review and meta-analysis that aims to assess the benefits and safety of acupuncture in patients with $\mathrm{BC}$.

Methods and analysis Clinical trials will be identified through nine databases from inception to December 2020, using Cochrane Central Register of Controlled Trials (CENTRAL), MEDLINE, Embase, Allied and Complementary Medicine Database (AMED), CINAHL, China National Knowledge Infrastructure (CNKI), Chinese Biomedical Literature Database (CBM), VIP Database and Wanfang Database. Search words will be used for the $\mathrm{BC}$ and acupuncture. The analysis would include randomised, controlled, clinical trials of adults with BC that were published in either Chinese or English. The primary outcome is to measure pain relief. Two or three reviewers should be in charge of study selection, data extraction and evaluating the risk of bias. RevMan software (V.5.4) will be used to perform the assessment of the risk of bias and data synthesis.

Ethics and dissemination Ethics approval will not be required for this review, as it will only involve the collection of literature previously published. The results of this metaanalysis will be disseminated in a peer-reviewed journal or relevant conference, through publication.

Trial registration number CRD42020167510.

\section{INTRODUCTION}

\section{Description of the condition}

Biliary colic (BC) is an episodic attack of severe pain for at least 20 to $30 \mathrm{~min}$ in the right upper abdominal quadrant, which even radiates to the right-back or shoulder. ${ }^{1} \mathrm{BC}$ occurs within a couple of hours after eating a meal. It is the most common manifestation of gallstones. ${ }^{2} \mathrm{BC}$ often accompanied by nausea, vomiting or fever, affects at least one-third of the adult population with gallstones. ${ }^{3} \mathrm{BC}$ may also occur after cholecystectomy, which is described as postcholecystectomy syndrome (PCS).

When a patient presents with BC, the most critical immediate phase is adequate to control the symptoms, including appropriate analgesia. Non-steroid anti-inflammatory
Strengths and limitations of this study

This study presents an explicit and replicable methodology for analysing the effect of acupuncture in order to provide a comprehensive synthesis of the evidence for patients with biliarycolic, clinicians and policymakers.

- Two or more reviewers will independently perform the study selection, data extraction and assessment of the risk of bias.

- The differences between acupuncture treatments could be a source of heterogeneity between the studies and could cause considerable heterogeneity in this meta-analysis.

- Since different scales may be used in trials, the pooling of analysis of all included studies may not be possible; however, subgroup analyses will be conducted according to different outcomes.

drugs (NSAIDs), opioid drugs and spasmolytic drugs have been widely used to relieve BC. NSAIDs are usually used as the first-line of treatment, such as diclofenac, ketoprofen and indomethacin. ${ }^{4}$ In patients with active peptic ulcer disease, recent gastrointestinal bleeding, or renal insufficiency, NSAIDs must be used with caution, however. At the same time, NSAIDs can increase the risk of severe side effects. ${ }^{5}$

\section{Description of the intervention}

Acupuncture as a non-pharmacological treatment is commonly considered a safe and effective treatment for a variety of conditions that cause pain and discomfort. ${ }^{6}$ Acupuncture has been reported to have a clinically relevant, lasting effect on chronic pain. ${ }^{7}$ In the meantime, acupuncture has a potential role in controlling acute pain, and it has a better safety profile. ${ }^{8-10}$ Acupuncture, as an adjunctive treatment, led to the decreased dosage of pain killers. ${ }^{11-13}$

\section{How the intervention might work}

Clinically, acupuncture affects the motility of the extrahepatic biliary tract, and improves the clinical symptom of BC. ${ }^{14}$ But the 
underlining mechanisms are still unknown. Zhao et al found that acupuncture has bidirectional effects on gallbladder pressure and Oddi's sphincter, which is possibly attributable to autonomic reflexes and somatovisceral interactions. ${ }^{16}$ Studies have observed the regulatory effect of acupuncture on peripheral and central neurotransmitters to discuss the possible mechanisms for the effect of analgesia, such as stimulating the release of endogenous opioid endorphin. ${ }^{17} 18$ Meanwhile, the acupunctureinvolving brain regions have been mapped over the past 20 years. $^{19}$

\section{Why it is important to perform this review}

In recent years, the number of clinical reports on acupuncture treatment for BC has increased gradually. ${ }^{15} 20$ So far, only one systematic review (SR) published in 2020 resulted that there is no statistical difference between acupuncture plus conventional medicine $(\mathrm{CM})$ and single $\mathrm{CM}$ in reducing pain for patients with $\mathrm{PCS} .{ }^{21}$ In this case, a lack of SR to address the following pivotal questions: (1) is acupuncture effective in treating biliary colic with gallstones and (2) whether acupuncture an effective adjunctive therapy to $\mathrm{CM}$ for $\mathrm{BC}$ with gallstones. Thus, there is a chance for us to conceive a comprehensive review to determine the efficacy and safety of acupuncture for BC.

\section{Objectives}

To assess the benefits and safety of acupuncture in patients with BC.

\section{METHODS}

\section{Criteria for including studies in this review}

Types of studies

Randomisedcontrolled trials (RCTs) reported in Chinese or English will be included. Others such as animal research, uncontrolled trials or case reports will be excluded.

\section{Types of participants}

All people who are presenting with $\mathrm{BC}$ with gallstones according to the definitions and diagnostic work-up in the individual trials.

\section{Types of interventions}

The acupuncture style considered must involve the insertion of needles at the acupuncture points. This study will include acupuncture therapy which is used alone or as an add-on to the conventional drug. At the same time, other types of acupuncture points without needle insertion into skin (such as laser, moxibustion or massage), will be excluded.

\section{Types of comparator(s)/control}

The control group will be considered and classified as following:

1. Acupuncture versus sham/minimal acupuncture or placebo;

2. Acupuncture versus conventional drugs/routine care;
3. Acupuncture with active treatment versus active treatment alone.

If the purpose of the study is to compare different forms of acupuncture or acupuncture with other complementary therapies for the treatment of BC, such studies will be excluded.

Types of outcome measures

\section{Primary outcomes}

Quantification of pain relief rated after acupuncture treatment in the trials on quantitative scales, such as Visual Analogue Scale.

\section{Secondary outcomes}

1. Number of people with cholelithiasis-related complications.

2. Quality of life.

3. Adverse events.

4. The time needed to obtain pain relief: complete pain relief or any small amount of pain relief (as defined by the different trials).

\section{Search methods for identification of studies \\ Electronics searches}

The following nine databases including Cochrane Central Register of Controlled Trials, MEDLINE, Embase, Allied and Complementary Medicine Database, CINAHL, China National Knowledge Infrastructure, Chinese Biomedical Literature Database, VIP Database and Wanfang Database will be searched from inception to December 2020.

The terms will be searched as following: biliary colic, biliary disease, biliary pain, biliary complication, acute cholecystitis, cholelithiasis-related complication, acupuncture, acupuncture therapy, manual acupuncture, electro-acupuncture, acupoint, auricular acupuncture and warm needling. The search strategy for MEDLINE is shown in table 1 . We would use the equivalent search words in Chinese databases.

\section{Searching other resources}

The following clinical trial registries will be used to retrieve ongoing trials: the NIH clinical registry ClinicalTrials.gov, the International Clinical Trials Registry Platform (ICTRP) and the Chinese clinical registry. We would retrieve the relevant SRs and meta-analyses manually and review it to identify additional studies. Useful but incomplete information would be acquired from the contact trial personnel.

\section{Data collection and analysis \\ Selection of studies}

According to the inclusion criteria, all retrieved studies will be assessed by reviewers (YZ and YC) in the light of titles and abstracts. Full text of the qualified study will be reviewed if necessary. A third reviewer (LH) will arbitrate if any disagreement occurs. For excluded studies, the exclusion reason will be listed. The procedure of study selection will be shown in a Preferred Reporting Items 


\begin{tabular}{|c|c|}
\hline No. & Search items \\
\hline$\# 1$ & randomized controlled trial (pt) \\
\hline \#2 & controlled clinical trial (pt) \\
\hline \#3 & randomized (tiab) \\
\hline \#4 & placebo (tiab) \\
\hline \#5 & clinical trials (MeSH) \\
\hline \#6 & randomly (tiab) \\
\hline$\# 7$ & trial (ti) \\
\hline \#8 & \#1 OR \#2 OR \#3 OR \#4 OR \#5 OR \#6 OR \#7 \\
\hline \#9 & humans (MeSH) \\
\hline \#10 & \#8 and \#9 \\
\hline$\# 11$ & biliary colic (MeSH) \\
\hline \#12 & cholecystitis (MeSH) \\
\hline \#13 & $\begin{array}{l}\text { (biliary disease or biliary pain or biliary complication } \\
\text { or acute cholecystitis or cholelithiasis-related } \\
\text { complication): ti,ab }\end{array}$ \\
\hline \#14 & $\# 11$ or \#12 or \#13 \\
\hline \#15 & acupuncture therapy (MeSH) \\
\hline \#16 & acupuncture (MeSH) \\
\hline$\# 17$ & $\begin{array}{l}\text { (acupuncture or body acupuncture or manual } \\
\text { acupuncture or electroacupuncture or electro- } \\
\text { acupuncture or acupoint or auricular acupuncture or } \\
\text { warm needling): ti,ab }\end{array}$ \\
\hline \#18 & $\# 15$ or \#16 or \#17 \\
\hline \#19 & $\# 10$ and $\# 14$ and \#18 \\
\hline
\end{tabular}

for Systematic Reviews and Meta-Analyses flow chart (figure 1).

\section{Data extraction and management}

Two reviewers (YZ and YC) will double-check and collect information from all qualifying studies and transferred into RevMan software (V.5.4). ${ }^{22}$ A predefined data acquisition form will be entering with details: author list and affiliation, publication source, country, interventions, outcomes, adverse effects and so on. The acupuncture intervention details (such as the type of acupuncture, number of needle insertions, depths of insertion, selection of points, de-qi, needle stimulation) will be elaborated according to STandards for Reporting Interventions in Clinical Trials of Acupuncture (STRICTA). ${ }^{23} \mathrm{~A}$ third reviewer (LH) will arbitrate if any discrepancy noticed. We would obtain the information which is not available by contacting the authors.

\section{Assessment of risk of bias}

A systematic review of each study for the bias risk will be done by two independent reviewers (NS and YZ) by using the Cochrane Handbook for Systematic Reviews of Interventions. Six domains which are the bias of selection, performance, detection, attrition, reporting and other sources, will be assessed. For each domain, trials

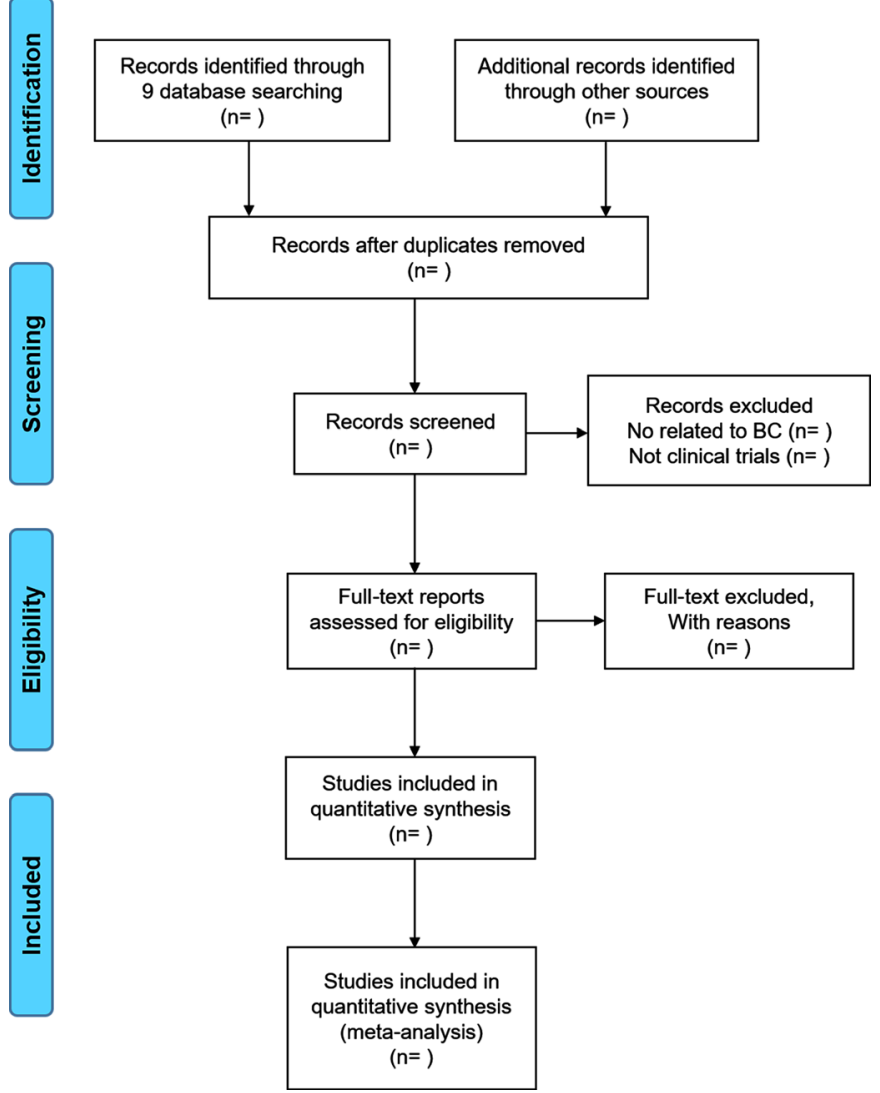

Figure 1 Flow diagram of the study selection process.

are going to be rated as low risk, high risk or unclear after evaluation. ${ }^{24}$ Involved authors will be contacted if anything unclear. Discrepancies will be arbitrated by a third reviewer $(\mathrm{LH})$ as well.

\section{Measures of treatment effect}

Synthesising and statistically analysing of the power data will use RevMan V.5.4. The dichotomous data will be analysed using a risk ratio with $95 \%$ CI. The mean difference or standard mean difference (SMD) with $95 \%$ CI will be used to analyse the continuous data. If different evaluation tools are used, SMD will be used.

\section{Unit of analysis issues}

The analysis will be based on the aggregated outcome data, which is caused by the lack of individual patient data.

\section{Dealing with missing data}

The authors will be contacted by reviewers to obtain the missing information which was not clearly described in each study included. ${ }^{25}$ If available, we will preferentially use the data from intention-to-treat analysis. ${ }^{26}$ If continuous outcome data is not expressed as means and SD, we will try to re-calculate means and SD as the first choice.

At the same time, if feasible, we will conduct a sensitivity analyses to address the potential impact of missing data. ${ }^{27}$ The potential impact of the effect of missing information on the final findings of the review will be addressed in the discussion. 


\section{Assessment of heterogeneity}

Forest plots will be used to present effect sizes and corresponding 95\% confidence limits for each study. At the same time, the inconsistency will be quantified by calculating the $\mathrm{I}^{2}$ test.

\section{Assessment of reporting biasses}

A funnel plot for observing the reporting biasses will be generated when more than 10 studies are included. ${ }^{25}$ Potential reporting biasses will be further assessed with Egger's test.

\section{Data synthesis}

Data synthesis will be performed through RevMan software (V.5.4). The random-effects model will be used to pool the data. When necessary, the possible causes of the large degree of heterogeneity will be analysed, or a subgroup analysis will be conducted. If the heterogeneity is considerable in the included trials, no meta-analysis will be performed.

\section{Subgroup analysis and investigation of heterogeneity}

Subgroup analysis will be conducted if data is available. Variations will be considered in the characteristics of the treatments for acupuncture, participants, control types. And there will be subgroups to interpret the heterogeneity.

\section{Sensitivity analysis}

The robustness of the main decisions made during the monitoring review process will be conducted through a sensitivity analysis. Several decision-making nodes for sensitivity review need to be considered in the system review process, such as methodological flaws, small research and data loss. Sensitivity analysis, as suggested in the Cochrane Handbook, includes two steps: in the first step, all major meta-analysis studies need to be included, and second, those studies that are known to be eligible. The results of the sensitivity analysis will be provided in the summary table. As shown by the results of the sensitivity analysis, the risk of bias will be discussed during the review process.

\section{Other analysis}

The use of meta-regression analysis will be considered to explore the potential heterogeneous resources.

\section{Summary of evidence}

The Grading of Recommendations Assessment, Development and Evaluation (GRADE) system approach will be used to measure the quality of main outcomes by two reviewers (NS and $\mathrm{YZ}$ ).$^{28}$ And the results will be present in 'Summary of findings' tables. The assessments of evidence quality will be rated 'high', 'moderate', 'low' or 'very low'. ${ }^{28}{ }^{29}$ Evidence of specific studies will be evaluated based on the risk of bias, inaccuracy, inconsistency, publication bias, indirectness, dose-response relation or effect size.
Patient and public involvement

No patient involved.

\section{Ethics and dissemination}

Ethics approval will not be required for this review, as it will only involve the collection of literature previously published. All included studies will be in accordance with the Declaration of Helsinki and current ethical standards. The results of this meta-analysis will be disseminated in a peer-reviewed journal or relevant conference, through publication.

\section{DISCUSSION}

There are currently several ongoing RCTs and many others completed of acupuncture for BC. This metaanalysis will provide a relatively convincing conclusion as to whether acupuncture is effective and safe for BC. We assume the findings taken from this study would have important consequences for people with BC, physicians and policymakers. If this protocol needs to be amended, we shall provide the date of each amendment and, therefore, state the reasons.

Contributors NS, F-RL and RS designed the systematic review. NS drafted the protocol. RS, F-RL and WZ revised the manuscript. NS, YZ, YC, SC, JZ, GX and LH participated in the design of data synthesis and analysis plan. All authors have read and approved the publication of the protocol.

Funding This study was supported by grants from the National Natural Science Foundation of China for the Youth (NO. 81904096), the major programme of the National Natural Science Foundation of China (NO. 81590950, 81590951), the China Postdoctoral Science Foundation (N0. 2019M653361), Research Open Project of Zhejiang Chinese Medical University (ZYX2018003), 'Xinglin Scholars' Subject Talent Research Promotion Plan of Chengdu University of Traditional Chinese Medicine (2019), and Special Project of "Central Government Guides Local Science and Technology Development" in Sichuan Provincial Department of Science and Technology (2020ZYD046).

\section{Competing interests None declared.}

Patient and public involvement Patients and/or the public were not involved in the design, or conduct, or reporting, or dissemination plans of this research.

Patient consent for publication Not required.

Provenance and peer review Not commissioned; externally peer reviewed.

Open access This is an open access article distributed in accordance with the Creative Commons Attribution Non Commercial (CC BY-NC 4.0) license, which permits others to distribute, remix, adapt, build upon this work non-commercially, and license their derivative works on different terms, provided the original work is properly cited, appropriate credit is given, any changes made indicated, and the use is non-commercial. See: http://creativecommons.org/licenses/by-nc/4.0/.

\section{ORCID iDs}

Ning Sun http://orcid.org/0000-0002-1064-5705

Yuanfang Zhou http://orcid.org/0000-0002-5848-6432

Fanrong Liang http://orcid.org/0000-0001-8518-9268

Ruirui Sun http://orcid.org/0000-0003-0555-2042

\section{REFERENCES}

1 Baiu I, Hawn MT. Gallstones and biliary colic. JAMA 2018;320:1612.

2 Fraquelli M, Casazza G, Conte D, et al. Non-Steroid antiinflammatory drugs for biliary colic. Cochrane Database Syst Rev 2016;9:CD006390.

3 Cervellin G, Mora R, Ticinesi A, et al. Epidemiology and outcomes of acute abdominal pain in a large urban emergency department: retrospective analysis of 5,340 cases. Ann Transl Med 2016;4:362. 
4 European Association for the Study of the Liver (EASL). Electronic address: easloffice@easloffice.eu. EASL clinical practice guidelines on the prevention, diagnosis and treatment of gallstones. $J$ Hepatol 2016;65:146-81.

5 Pepine CJ, Gurbel PA. Cardiovascular safety of NSAIDs: additional insights after precision and point of view. Clin Cardiol 2017:40:1352-6.

6 World Health Organization. Acupuncture: review and analysis of reports on controlled clinical trials, 2002.

7 Vickers AJ, Vertosick EA, Lewith G, et al. Acupuncture for chronic pain: update of an individual patient data meta-analysis. $J$ Pain 2018;19:455-74.

8 Grissa $\mathrm{MH}$, Baccouche $\mathrm{H}$, Boubaker $\mathrm{H}$, et al. Acupuncture vs intravenous morphine in the management of acute pain in the ED. Am J Emerg Med 2016;34:2112-6.

9 Tsai S-L, Fox LM, Murakami M, et al. Auricular Acupuncture in Emergency Department Treatment of Acute Pain. Ann Emerg Med 2016;68:583-5

10 Kaynar M, Koyuncu F, Buldu İbrahim, et al. Comparison of the efficacy of diclofenac, acupuncture, and acetaminophen in the treatment of renal colic. Am J Emerg Med 2015;33:749-53.

11 Zheng Z, Gibson S, Helme RD, et al. Effects of electroacupuncture on opioid consumption in patients with chronic musculoskeletal pain: a multicenter randomized controlled trial. Pain Med 2019;20:397-410.

12 Deng G, Giralt S, Chung DJ, et al. Reduction of opioid use by acupuncture in patients undergoing hematopoietic stem cell transplantation: secondary analysis of a randomized, shamcontrolled trial. Pain Med 2020;21:636-42.

13 Crawford P, Penzien DB, Coeytaux R. Reduction in pain medication prescriptions and self-reported outcomes associated with acupuncture in a military patient population. Med Acupunct 2017;29:229-31.

14 CB L, Zhu JM, Huang JP. Pain-Relieving effects of acupuncture at Yanglingquan and Dannang acupoints in acute cholecystitis. Shanghai Journal of Traditional Chinese Medicine 2011;45:56-7.

15 Qiu K, Jing M, Sun R, et al. The status of the quality control in Acupuncture-Neuroimaging studies. Evid Based Complement Alternat Med 2016;2016:1-14
16 Zhao J, Yu Y, Luo M, et al. Bi-Directional regulation of acupuncture on extrahepatic biliary system: an approach in guinea pigs. Sci Rep 2017;7:14066.

17 Goldman N, Chen M, Fujita T, et al. Adenosine A1 receptors mediate local anti-nociceptive effects of acupuncture. Nat Neurosci 2010;13:883-8

18 Ali U, Apryani E, Ahsan MZ, et al. Acupuncture/Electroacupuncture as an alternative in current opioid crisis. Chin $\mathrm{J}$ Integr Med 2020;26:643-7.

19 Jiang HF, Zhao C, Tang XF. Clinical analgesic effect of acupuncture at GB34 (Yanglingquan) and EX-LE6 (Dannang) for biliary colic caused by acute cholecystitis. J Hepatopancreatobiliary Surg 2016;28:481-3.

20 Feng LS, CF G, LH Y. Pain-Relieving effects of acupuncture in treating 260 patients with acute Cholecystalgia. Journal of Yunnan University of Traditional Chinese Medicine 2014;1:43-4.

21 Yin Z, Xiao Q, Xu G, et al. Acupuncture for the Postcholecystectomy syndrome: a systematic review and meta-analysis. Evid Based Complement Alternat Med 2020;2020:1-19.

22 Collaboration RTC. Review manager (RevMan). 5.0. Copenhagen: The Nordic Cochrane Collaboration, 2008.

23 MacPherson H, Altman DG, Hammerschlag R, et al. Revised standards for reporting interventions in clinical trials of acupuncture (stricta): extending the CONSORT statement. J Evid Based Med 2010;3:140-55.

24 Higgins JPT, Altman DG, Gøtzsche PC, et al. The Cochrane collaboration's tool for assessing risk of bias in randomised trials. BMJ 2011;343:d5928.

25 Higgins JP, Green S. Cochrane Handbook for systematic reviews of interventions. Wiley Online Library, 2008.

26 Abraha I, Cozzolino F, Orso M, et al. A systematic review found that deviations from intention-to-treat are common in randomized trials and systematic reviews. J Clin Epidemiol 2017;84:37-46.

27 Porta N, Bonet C, Cobo E. Discordance between reported intentionto-treat and per protocol analyses. J Clin Epidemiol 2007;60:663-9.

28 Guyatt GH, Oxman AD, Vist GE, et al. Grade: an emerging consensus on rating quality of evidence and strength of recommendations. $B M J$ 2008;336:924-6.

29 Guyatt GH, Oxman AD, Schünemann HJ, et al. Grade guidelines: a new series of articles in the Journal of clinical epidemiology. J Clin Epidemiol 2011;64:380-2. 